\title{
A Friction Control Strategy for Shock Isolation
}

\author{
Mohd Ikmal Ismail, Neil Ferguson \\ Institute of Sound and Vibration Research, University of Southampton, Southampton, United Kingdom \\ Email address: \\ mii1m13@soton.ac.uk (M. I. Ismail),nsf@isvr.soton.ac.uk (N. Ferguson)
}

\section{To cite this article:}

Mohd Ikmal Ismail, Neil Ferguson. A Friction Control Strategy for Shock Isolation. International Journal of Mechanical Engineering and Applications. Vol. 7, No. 3, 2019, pp. 78-90. doi: 10.11648/j.ijmea.20190703.12

Received: May 25, 2019; Accepted: June 10, 2019; Published: August 10, 2019

\begin{abstract}
A control strategy is presented incorporating friction which can be adapted within a cycle of vibration. During base shock input, the friction is switched on and off based on specified response parameters. The predicted response of a semi active system is compared with that of a passive isolation system. The strategy is shown to produce an improved displacement reduction and a smaller maximum displacement compared to the base input; a result which cannot be obtained with a typical passive system. The models are then validated using an experimental rig, representing a two degree of freedom system, having an electromagnet to switch on and off friction via the control logic. Good agreement is obtained in addition to identifying optimum parameter choices.
\end{abstract}

Keywords: Shock Isolation, Semi Active Friction, Two Degree of Freedom Model

\section{Introduction}

A linear vibration or shock isolator is often used, typically comprising an elastic element acting in parallel with a damping element. Ismail and Ferguson [1] utilised dry friction to isolate a simple system undergoing shock excitation and noted some advantages of dry friction over a viscous damping element. However, passive isolation systems have limitations because the choice of the constant system properties produce good characteristics and subsequent shock behaviour only for limited ranges. Ismail [2] found that in a passive system incorporating friction, the direction of the friction force changes with respect to the relative sliding velocity. This can be detrimental in some situations because the forces can act in the same direction as the shock input.

To further improve the response of a system subjected to shock, a friction control strategy for isolation can be introduced. Lu et al [3] stated that numerous control methods exist in the literature for variable friction devices, which can generally be classified as either discontinuous or continuous force control. Among these, semi active and adaptive controls are widely used because of great advantages in terms of low power consumption compared to active control, being normally stable with no additional energy supplied. Ramirez [4] and Liu Y et al [5] mentioned that the difference between the semi active and adaptive configurations is that in the adaptive systems the properties are changed relatively slowly (continuous), while in the semi active systems the properties are changed within a cycle of vibration (discontinuous).

Because semi active control offers great advantages, there are a significant number of studies related to this type of control. Jalili [6] summarized different types of semi active control ranging from variable rate dampers, variable spring elements, hybrid elements and on-off control. The study of sequential variations existed as early as 1974 with the study performed by Karnopp et al [7] and subsequently a significant number of studies have proposed semi active vibration isolation employing on-off damping. On-off control is popular as its implementation is very simple, producing significant response reduction. Implementation is typically based on the sign of the response and input parameters and not their magnitude. Most studies focus on implementing control for a linear system subjected to a harmonic input. For example, Dupond et al [8] proposed control laws for friction dampers which maximize the energy dissipation in an instantaneous sense by modulating the normal force at the friction interface.

There are fewer papers discussing control using friction for shock isolation, such as Karnopp et al [7], Ferri and Heck [9] and Yamaguchi and Yashima [10]. Semi active control of the normal force has previously been used to control the friction force acting on the structure. For example, Ferri and Heck [9] 
presented three control strategies for a low bandwidth friction system to obtain excellent shock isolation properties. Yamaguchi and Yashima [10] presented the strategy of on-off control for a friction force at a spring support. The system comprised a primary isolated mass supported by a primary spring connected to a secondary mass supported by a secondary spring via an interfacial friction force.

More recently, there has been interest in the effect of purely passive friction within the isolators themselves; where the approach has often been experimental and typically the isolators comprise wire rope that is involved in the design of potentially quite nonlinear isolation components (see Ledezma-Ramirez et al [17] and Guzman-Nieto et al [18] and Tapia-Gonzalez [19]). The approaches have centred on the design of quasi zero (dynamic) stiffness devices, suitable for carrying static load but in a dynamic situation having low stiffness and having high dissipation due to the friction acting. For such systems the mathematical models can no longer be considered by simple mass-spring-viscous damper elements but more general Bouc-Wen models [17].

A complementary area which using friction but in a switchable mode, not in an attached tuned absorber is the work in the area of the so-called smart-spring system ([20-21]). It typically comprises primary and secondary spring acting in parallel, but the secondary spring has one end that can actively (by piezoelectric actuator) be either free or in sliding contact when actuated. De Ni et al work [20] concentrated on a

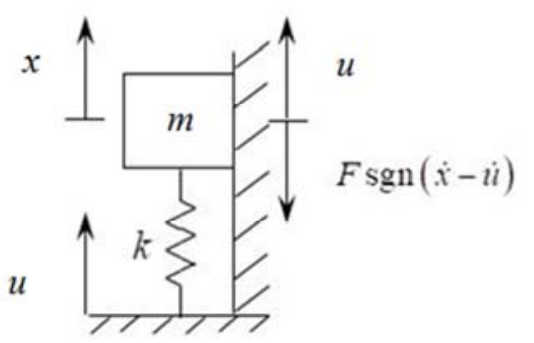

deriving a frequency response function for such a system, whilst the work by Gustavo Rocha Vieira et al [21] gave free and forced response predictions, but not applied it to the isolation application as considered herein. This paper presents and discusses friction control strategies for two degree of freedom systems. Various control strategies were developed from the idea of reducing the net force acting on the isolated mass during the base input. The predicted response were then compared to the corresponding passive system response reported by Ismail and Ferguson [1]. Subsequently, an electromagnetic control system was designed and implemented. This was incorporated into an existing passive system rig developed by Ismail [2] having the capability to switch on and off friction via the identified control logic. The revised test rig was subsequently used to validate the response of a system with the proposed control strategies.

\section{Benchmark Single Degree of Freedom System with Semi Active Friction}

\subsection{Control Strategy}

Figure 1 is the idealised configuration and free body diagram of a single degree freedom system, showing two forces acting on the isolated mass, namely the spring stiffness force and the Coulomb sliding friction force.

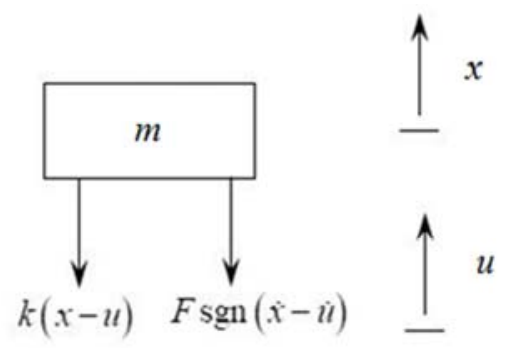

Figure 1. Single degree of freedom system configuration and the accompanying free body diagram of a single degree of freedom system with friction, $u=$ base input displacement, $F=$ sliding friction force, $m=$ the isolated mass, $k=$ support stiffness.

When the friction acts in the opposite direction to the spring force and the mass velocity the friction is switched on to reduce the acceleration of the mass. However, when the friction acts in the same direction as the spring force and the mass velocity, the friction is switched off to avoid the addition of the forces and subsequently an increase in the net mass acceleration. An almost identical control strategy has been used by Stammers and Sireteanu [11] and Guglielmino et al (2004) [12]. Stammers and Sireteanu [11] applied different strategies for forced and free vibration of the

$$
\begin{array}{ll}
\hat{F}=0 & \text { if } \dot{x} \geq 0, x-u<0 \text { and } \dot{x}-\dot{u}<0 \\
& \text { or } \dot{x}<0, x-u \geq 0 \text { and } \dot{x}-\dot{u} \geq 0 \\
\hat{F}=\widehat{F} \text { on } & \text { For other conditions }
\end{array}
$$

$\widehat{F_{\text {on }}}$ is the value of the friction force when the friction is turned on. This constant value can be chosen to achieve the best isolated controlled response. system. The best characteristic of a controlled friction system is that the friction can be turned off completely, which is not the case for a controlled viscous damper system as described by Liu Y et al [5].

The control strategy is modified from the original concept widely published by including an additional parameter namely the mass velocity to perform the logic, as presented in Equation 1. Base input $u(t)$ is a versed sine with pulse length $T p$ similar to the previous study by Ismail and Ferguson [1].

$$
\begin{aligned}
& \text { for } 0 \leq t \leq T_{p} \\
& \text { (during the base shock input) } \\
& \text { for } 0 \leq t \leq T_{p} \\
& \text { (during the base shock input) }
\end{aligned}
$$

Implementation can be performed accurately, since the direction of the spring and friction forces can be determined at every instant of time by measuring both the relative 
displacement and isolated mass absolute velocity. The possible issue with the control implementation is the discontinuity in the acceleration of the mass and the subsequent chattering at the switching points. Chatter is discussed in various papers written by Liu Y et al [5], Dupont et al [8] and Ahmadian et al [13], with discussion on how to avoid this. This is why a two degree of freedom system arrangement is preferable and its control strategy is presented in Section 3.

\subsection{Benchmark System Response}

Non-dimensional parameters are defined and used to describe the system responses. These are the nondimensional displacement $y=\frac{\omega_{n}^{2} x}{g}$, the non-dimensional friction force $\hat{F}=\frac{F}{m g}$, the non-dimensional base input $U=\frac{\omega_{n}^{2} u}{g}$, the non-dimensional time $\tau=\omega_{n} t$ and differentiation with respect to non-dimensional time $y^{\prime}=\frac{d y}{d \tau}$, $y^{\prime \prime}=\frac{d^{2} y}{d \tau^{2}}$.

For comparison with the respective passive system, the pulse length is chosen to be equal to the natural period of the system. The passive system response is amplified when the pulse length is equal or close to the natural period of the system as shown by Ismail [2]. The lowest maximum response is achieved when the non-dimensional friction value applied $\hat{F}=\frac{F}{m g}$ is approximately equal to 4 . The normalised displacement with respect to the input magnitude for this case is approximately unity, which means no isolation can be achieved with the passive system.

The comparison between the maximum displacement of a system with the proposed control strategy (dashed line), the control strategy widely used in the literature (dash dot line) and the corresponding passive system (solid line) is shown in Figure 2. The proposed control strategy is better than that previously published which produces improvement for small friction. No difference can be observed at higher friction forces, because the mass then sticks to the friction interface.

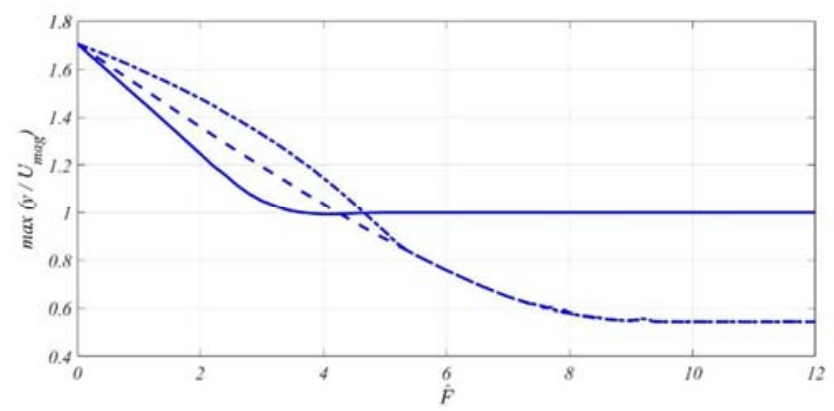

Figure 2. Comparison between the maximum response of a passive single degree of freedom system and a system with control under a versed sine base input when the friction force is varied according to Equation 1. Solid line: passive system. Dash line: controlled system introduced in this paper. Dash dot line: controlled system widely published in the literature [11, 12]. Nondimensional base input magnitude $U_{\text {mag }}=10$, pulse length $T_{p}=T$ and non-dimensional friction $\hat{F}=\frac{F}{m g}$.
The proposed control strategy can achieve a maximum displacement smaller than the input magnitude and the corresponding passive system when the non-dimensional friction force is larger than 4.3. There is no improvement achieved at lower magnitudes of the friction force with respect to the corresponding passive system. At higher friction, the passive system fully sticks to the friction interface, but the control system has a smaller maximum response. For non-dimensional friction force $\hat{F} \geq 10$, a normalised displacement $\frac{y}{U_{m a g}}=0.55$ was produced.

\section{Shock Response of a Two Degree of Freedom System with Semi Active Friction}

\subsection{Shock Strategy}

In a passive two degree of freedom system, previously introduced by Ismail and Ferguson [1], friction is applied to a secondary mass which is attached to the primary isolated mass by a secondary spring. Ismail and Ferguson [1] found that the instantaneous change during the start of the motion and every time friction changes its direction can be improved with a two degree of freedom configuration as friction is not applied directly to the primary mass. There is potential that applying the control strategy concept to reduce the net force acting on the primary mass could further reduce its displacement in a two degree of freedom configuration.

The free body diagram of a two degree freedom system is shown in Figure 3. Two forces acting on the primary mass; the forces from the primary and the secondary springs. The force from the secondary spring is dependent upon the motion of the secondary mass $x_{2}$, which is itself dependent upon the friction force. The idea is to control on and off friction, to subsequently cause the force from the secondary spring to oppose the force from the primary spring so as to minimise the net force acting on the primary mass.
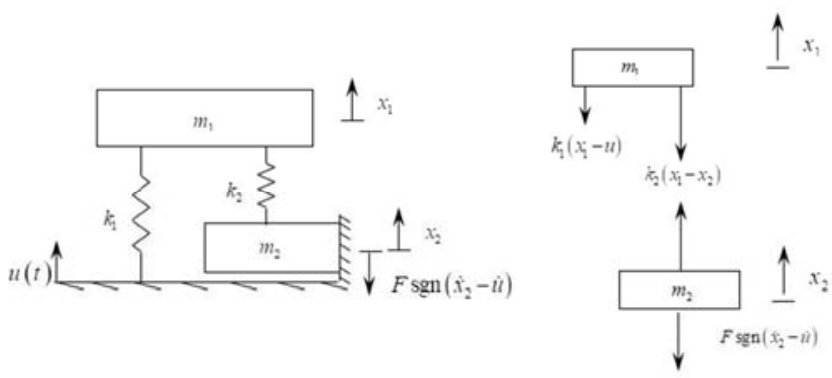

Figure 3. Two degree of freedom system configuration and the accompanying free body diagram of a two degree of freedom system with friction, $u=$ base input displacement, $F=$ sliding friction force, $m_{1}=$ primary isolated mass, $k_{1}=$ primary stiffness, $m_{2}=$ secondary mass, $k_{2}=$ secondary stiffness.

When a shock input is applied to the system, the primary spring is initially compressed and both masses move in the positive direction. In this instance, the secondary spring should be stretched to minimise the net force acting on the primary mass and this is only possible when there is no 
friction. The presence of friction during this instance will cause the compression of the secondary spring and an additional force applied to the primary mass. Assuming a Coulomb model for the sliding friction, its value depends upon the sign of the relative velocity between the secondary mass and the base input. During this initial stage, the velocity of the secondary mass is always smaller than the base input velocity which causes a compression of the secondary spring.

After some time, the relative velocity starts to become positive and friction will stretch the secondary spring. Because the primary spring is still in compression and the primary mass still moves in the positive direction, the presence of friction at this stage is necessary to reduce the net force acting on the primary mass. As the motion progresses, the primary spring force (relative displacement across the primary spring), the direction of friction (the sign of relative velocity between the secondary mass and the base input) and the primary mass velocity are recognised as three required parameters to implement the decision logic in order to reduce the primary mass displacement. Friction is only switched on when the primary spring and friction forces have opposite sign or when friction opposes the direction of primary mass motion. The direction of the secondary spring force is not considered, since the control strategy outlined appears to be sufficient to cause the force across the secondary spring to oppose the force across the primary spring most of the time. This control is presented mathematically as follows in Equation 2:

$$
\begin{array}{lll}
\hat{F}=0 & \text { if } \dot{x}_{1} \geq 0, x_{1}-u<0 \text { and } \dot{x}_{2}-\dot{u}<0 & \text { for } 0 \leq t \leq T_{p} \\
& \text { or } \dot{x_{1}}<0, x_{1}-u \geq 0 \text { and } \dot{x_{2}}-\dot{u} \geq 0 & \text { (during the base shock input) } \\
\hat{F}=\widehat{F} \text { on } & \text { For other conditions } & \text { for } 0 \leq t \leq T_{p} \\
& & \text { (during the base shock input) }
\end{array}
$$

$\widehat{F_{\text {on }}}$ is the value of the friction force when the friction is turned on.

\subsection{The Stiffness Ratio Effect on the System Response}

Another set of non-dimensional parameters are defined for and used for this configuration to describe the system responses. These are the two non-dimensional displacements $y_{1}=\frac{\omega_{1}^{2} x_{1}}{g}$ and $y_{2}=\frac{\omega_{2}^{2} x_{2}}{g}$, the frequency ratio $\widehat{\omega}=\frac{\omega_{2}}{\omega_{1}}$ where $\omega_{1}=\sqrt{\frac{k_{1}}{m_{1}}}$ and $\omega_{2}=\sqrt{\frac{k_{2}}{m_{2}}}$, the mass ratio $\mu=\frac{m_{2}}{m_{1}}$, the stiffness ratio $\hat{k}=\frac{k_{2}}{k_{1}}$, the non-dimensional friction force $\widehat{F}=\frac{F}{m_{1} g}$, the non-dimensional base input $U=\frac{\omega_{1}^{2} u}{g}$, the nondimensional time $\tau=\omega_{1} t$ and differentiation with respect to non-dimensional time $y^{\prime}=\frac{d y}{d \tau}, y^{\prime \prime}=\frac{d^{2} y}{d \tau^{2}}$ are as previously.

Figure 4 shows the maximum response of the primary mass of a two degree of freedom system under a versed sine base input with the control strategy implemented as described when the stiffness ratio $\hat{k}$ is varied and the friction force $\widehat{F}=2.5,5,7.5$ and 10 .

From Figure 4, the maximum displacement of the primary mass decreases with increased secondary stiffness up to certain point. Above this point, further increased secondary stiffness does not reduce the maximum primary mass displacement. By setting the stiffness ratio $\hat{k}$ at an intermediate value, the smallest maximum displacement could be achieved with a smooth acceleration at switching. This behaviour is similar to the passive system response as found by Ismail and Ferguson [1].

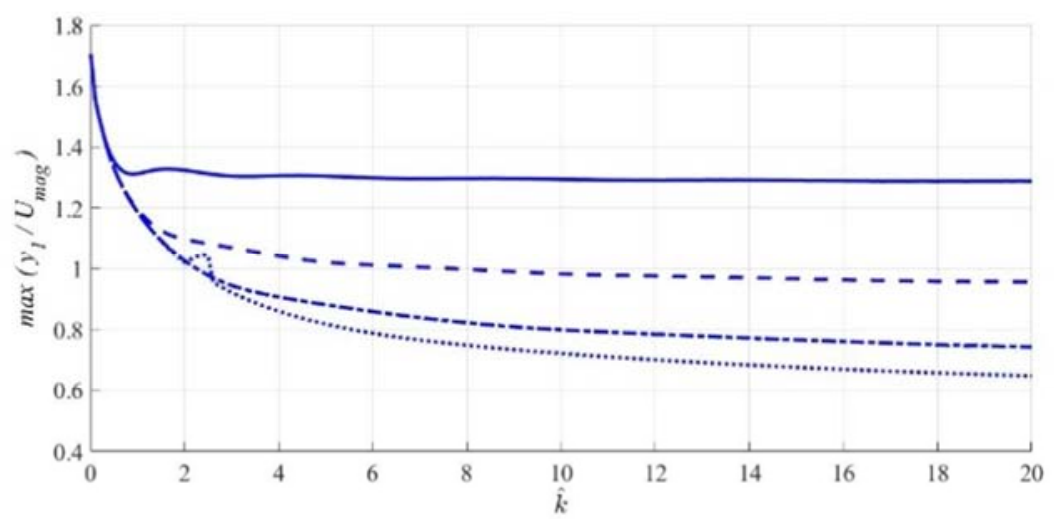

Figure 4. The maximum displacement response of the primary mass of a two degree of freedom system under a versed sine base input with control for different values of the stiffness ratio $\hat{k}$. Solid line: $\hat{F}=2.5$, Dash line: $\hat{F}=5$, Dash dot line: $\hat{F}=7.5$, Dot line: $\hat{F}=10$. Non-dimensional base input magnitude $U_{\text {mag }}=10$, pulse length $T_{p}=T_{1}$.

For very small stiffness ratio, $\hat{k}<0.6$, the maximum displacement of the primary mass does not change when friction is increased from $\hat{F}=2.5$ to $\hat{F}=10$. A soft secondary spring corresponds to a small stiffness ratio $\hat{k}$, friction does not have a significant effect on the response of the primary mass since it is applied to the secondary mass. For a higher stiffness ratio, the friction starts to show a significant effect on the primary mass displacement. From Figure 4 , the case of $\widehat{F}=5, \widehat{F}=7.5$ and $\hat{F}=10$ produce maximum normalised displacements smaller than unity when 
the stiffness ratio is sufficiently large i.e. it produces isolation. $\hat{F}=10$ can produce a normalised displacement lower than 0.6 , which is sufficiently close to the smallest response produced by a single degree of freedom system discussed in Section 2.2. This is when the stiffness ratio increases to higher than 37.2.

Table 1. Maximum normalized displacement of the primary mass with increment in the stiffness ratio $\widehat{k}$.

\begin{tabular}{|c|c|c|c|}
\hline \multirow[t]{2}{*}{ Non-dimensional friction force amplitude } & \multicolumn{3}{|c|}{ Maximum normalised displacement of the primary mass, $\frac{\max \left(y_{1}\right)}{U_{\operatorname{mag}}}$ for stiffness ratio $\widehat{k}$} \\
\hline & $\widehat{\boldsymbol{k}}=2$ & $\widehat{k}=10$ & $\widehat{\boldsymbol{k}}=\mathbf{8 0}$ \\
\hline$\widehat{F}=2.5$ & 1.32 & 1.29 & 1.28 \\
\hline$\widehat{F}=5$ & 1.10 & 0.98 & 0.92 \\
\hline$\hat{F}=7.5$ & 1.03 & 0.8 & 0.67 \\
\hline$\hat{F}=10$ & 1.02 & 0.72 & 0.57 \\
\hline
\end{tabular}

Three stiffness ratios were chosen to show the effect on the primary mass displacement and are summarised in Table 1.

\subsection{Time Response for the Best Case of Higher Stiffness Ratio}

The displacement of a two degree of freedom system under a versed sine base input with control and the comparison with passive system is shown in Figure 5. The maximum normalised displacement of the primary mass is shown in Table 2.

Bold line: base input, solid line: $\widehat{F}=2.5$, dash line: $\widehat{F}=5$, dash dot line: $\hat{F}=7.5$, dot line: $\hat{F}=10$. Non-dimensional base input magnitude $U_{\text {mag }}=10$, pulse length $T_{p}=T_{1}$, high stiffness ratio $\widehat{k}=80$. (a) Displacement of the primary mass with the control strategy suggested. (b) Displacement of the primary mass for a passive system.

Table 2. Comparison between the maximum normalized displacement of the primary mass for the system with control and the respective passive system. The case of large stiffness ratio $\widehat{k}=80$.

\begin{tabular}{|c|c|c|c|}
\hline \multirow{2}{*}{ Non-dimensional friction force amplitude } & \multicolumn{3}{|c|}{ Maximum normalised displacement of the primary mass, $\frac{\max \left(y_{1}\right)}{U_{m a g}}$} \\
\hline & With control strategy & Passive system & Percentage reduction \\
\hline$\widehat{F}=2.5$ & 1.28 & 1.17 & -9.4 \\
\hline$\hat{F}=7.5$ & 0.67 & 1 & 33 \\
\hline$\hat{F}=10$ & 0.57 & 1 & 43 \\
\hline
\end{tabular}

(a)

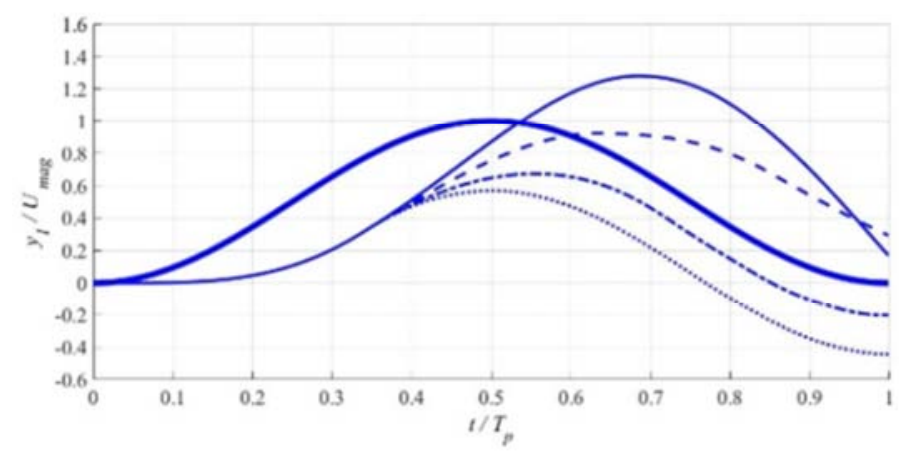

(b)

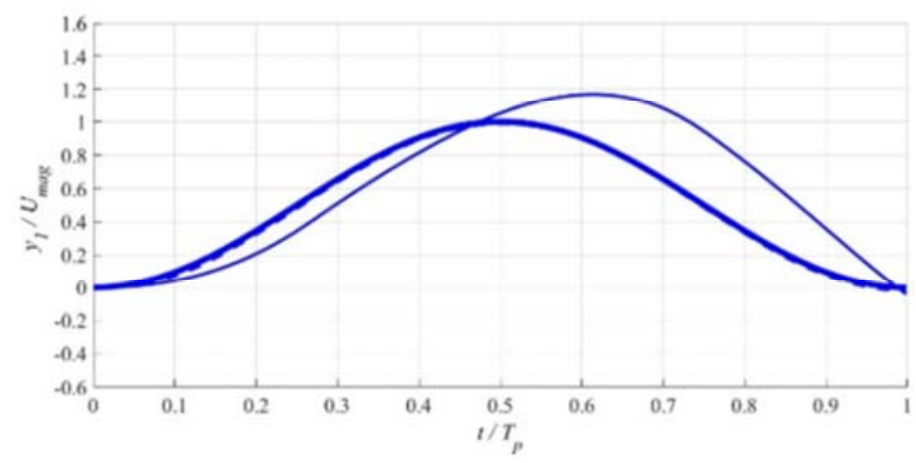

Figure 5. Displacement response of a two degree of freedom system under a versed sine base input with control and the comparison with its respective passive system. 
From Section 2.2, the predicted displacement of a single degree of freedom system with control is larger than the passive system for low levels of friction. This behaviour was not observed for the case of a soft secondary spring. In the case of an intermediate spring, this behaviour is manifested again because of the increased coupling between the primary mass and friction. In this case, when $\widehat{F}=2.5$, the maximum displacement of the primary mass is greater than the respective passive system (Table 2). The maximum displacement of the primary mass with control strategy experiences signifnt reduction compared to the passive system with increased levels of friction. The reduction is up to $43 \%$ when $\hat{F}=10$ with a maximum normalised displacement of the primary mass equal to 0.57 , comparable to the smallest maximum normalised displacement achieved by a single degree of freedom system in Section 2.2. Overall, for all of the higher friction values used the primary mass displacement is less than the input amplitude.

Figure 6 shows the primary mass velocity and acceleration. The transition at the switching points is sharper and higher frequency than the case of soft spring, but still relatively smooth without discontinuities, so showing the advantage of the additional single degree of freedom added system.

(a)

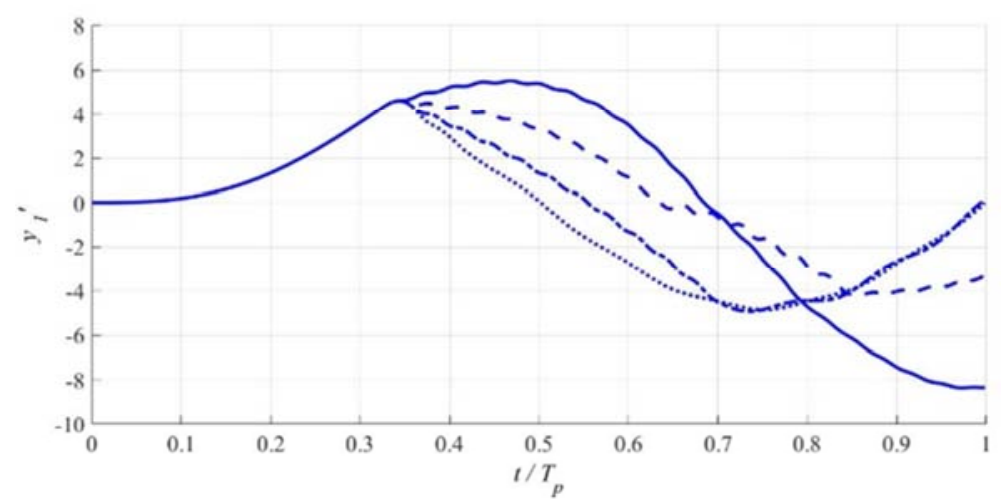

(b)

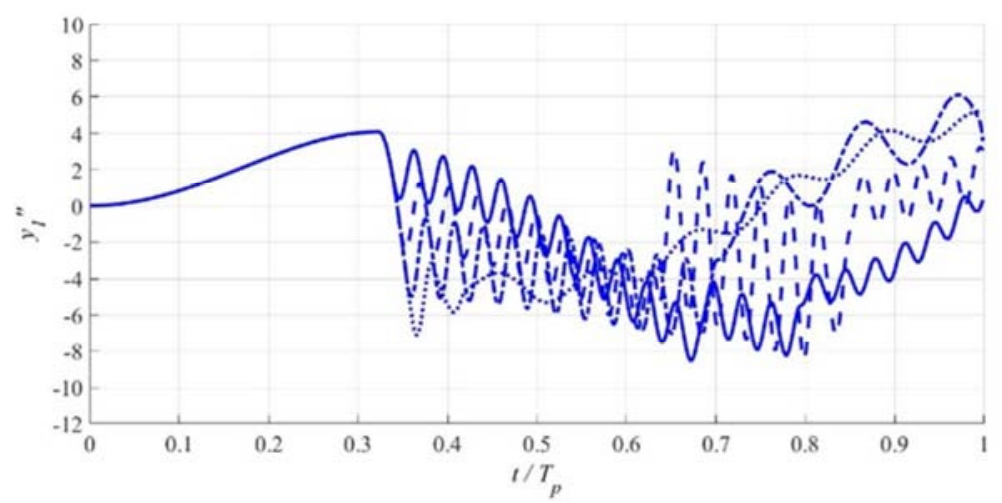

Figure 6. Primary mass response of a two degree of freedom system under a versed sine base input with control. Solid line: $\hat{F}=2.5$, dash line: $\hat{F}=5$, dash dot line: $\hat{F}=7.5$, dot line: $\hat{F}=10$. Non-dimensional base input magnitude $U_{\text {mag }}=10$, pulse length $T_{p}=T_{1}$, high stiffness ratio $\widehat{k}=80$. (a) Velocity of the primary mass. (b) Acceleration of the primary mass.

\section{Experimental Validation by Implementation of Switchable Friction}

\subsection{System Design}

An experimental switchable friction system should be able to switch on-off very quickly in real time to successfully replicate the predicted behaviour. A similar test rig was used as in the passive case performed by Ismail and Ferguson [1]. The main component is a $\mathrm{T}$ beam comprising two aluminium beams, namely a primary and a secondary beam which are connected with a bolted connection. A steel beam was used to apply friction to the ends of the secondary beam, which was bolted to the base, so that the friction interface could move with the base input following the model. A small screw at each end of the secondary beam was used to adjust the normal force between the ends of the secondary beam and the steel beam. In order to estimate the value of friction, a free vibration test was performed on the $\mathrm{T}$ beam system before the experimental validation. The level of friction was estimated by least square error fitting free vibration results to an analytical solution for any cycle of vibration. The experimental rig was suspended with cords and a shaker (Derritron VP4) below the base applied a base input. An electromagnet near to each end of the secondary beam controls on and off friction based on the logic developed. An electromagnet has been used in the past by Ramirez [4] and 
Agrawal et al $[14,15]$, and its instantaneous response has been recognised.

The proposed control strategy in Section 3.1 was used. A steel beam applies a normal load to both ends of the secondary beam, so friction is applied when the electromagnet is switched off. When the electromagnet is switched on, the electromagnet attracts the steel beam and both ends of the secondary beam are free to move without friction. The three parameters used for the control logic are the relative displacement between the end of the primary beam and the base, the relative velocity between the end of the secondary beam and the base (friction direction) and the absolute velocity at the end of the primary beam. This represents the relative displacement of the primary spring, relative velocity between the secondary mass and the base (friction direction) and the absolute velocity of the primary mass respectively. The friction is switched off by switching the electromagnet on when the relative displacement between the end of the primary beam and the base has the same sign as the relative velocity between the secondary beam and the base but the opposite sign to the absolute velocity of the end of the primary beam. In any other instants, the electromagnet is switched off to turn friction on. The outline of control decision is shown in Figure 7.

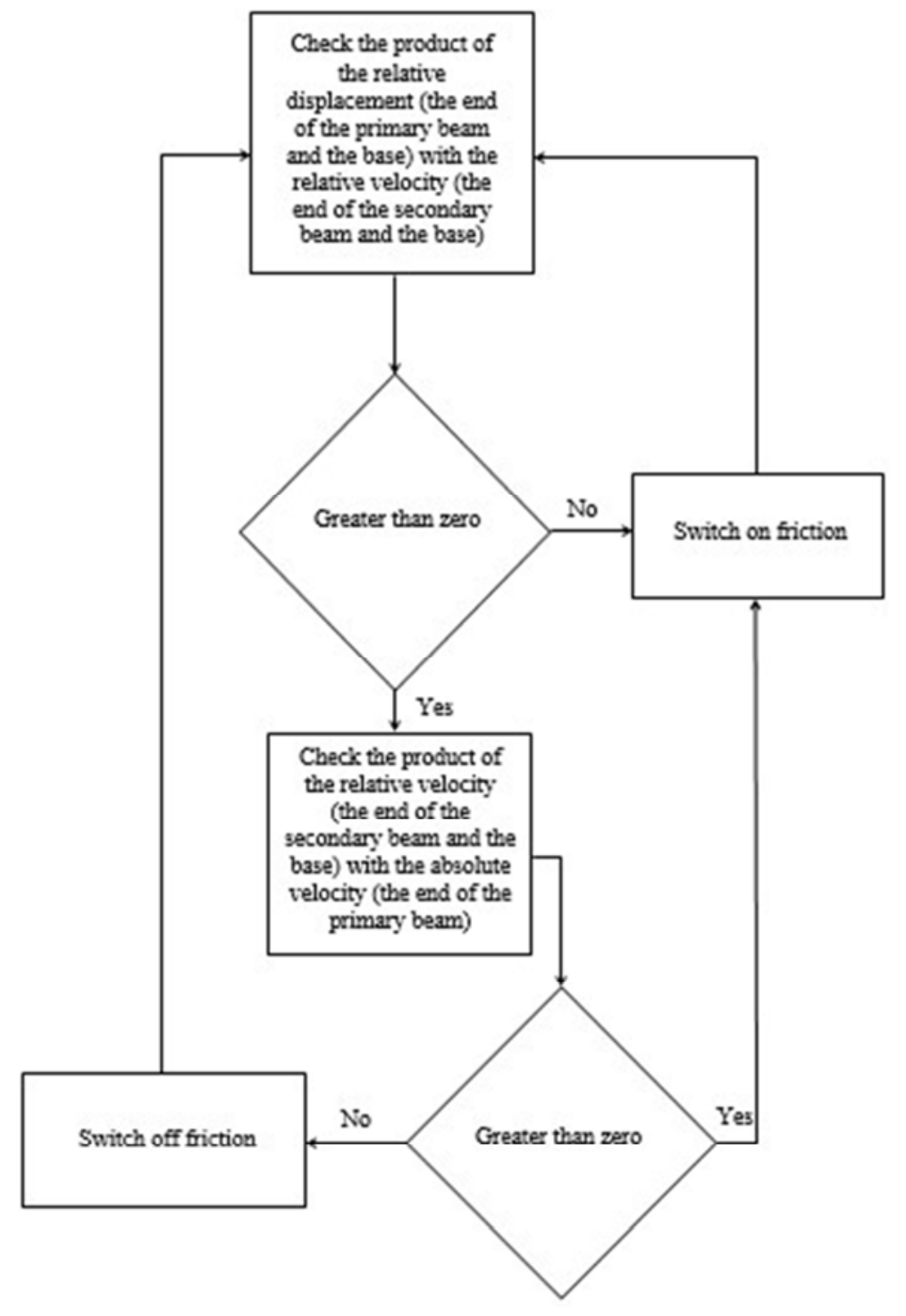

Figure 7. The decision logic to switch on and off friction depending on three measured parameters.

The control logic was implemented in the Simulink environment running real time on a D Space RTI 1005 platform. The acceleration signals from the accelerometers were integrated to obtain velocity and displacement. The absolute or relative acceleration was fed into a series of 'Discrete Time Integrator" blocks in Simulink to perform the integration. High pass filters first eliminate low frequencies before integration. The trigger function is included in the
Simulink code to perform the decision logic after the acceleration reaches a certain threshold.

The control action operates a relay which turns on and off the current from the power source to drive the electromagnet. The electromagnet cannot be directly connected to the D Space system due to output current limitation as stated by Jalili and Ramaratnam [16]. A metal-oxide semiconductor field-effect transistor (MOSFET) used for amplifying and 
switching electronic signals was used to implement this requirement. A schematic diagram of the experimental setup

is shown in Figure 8.

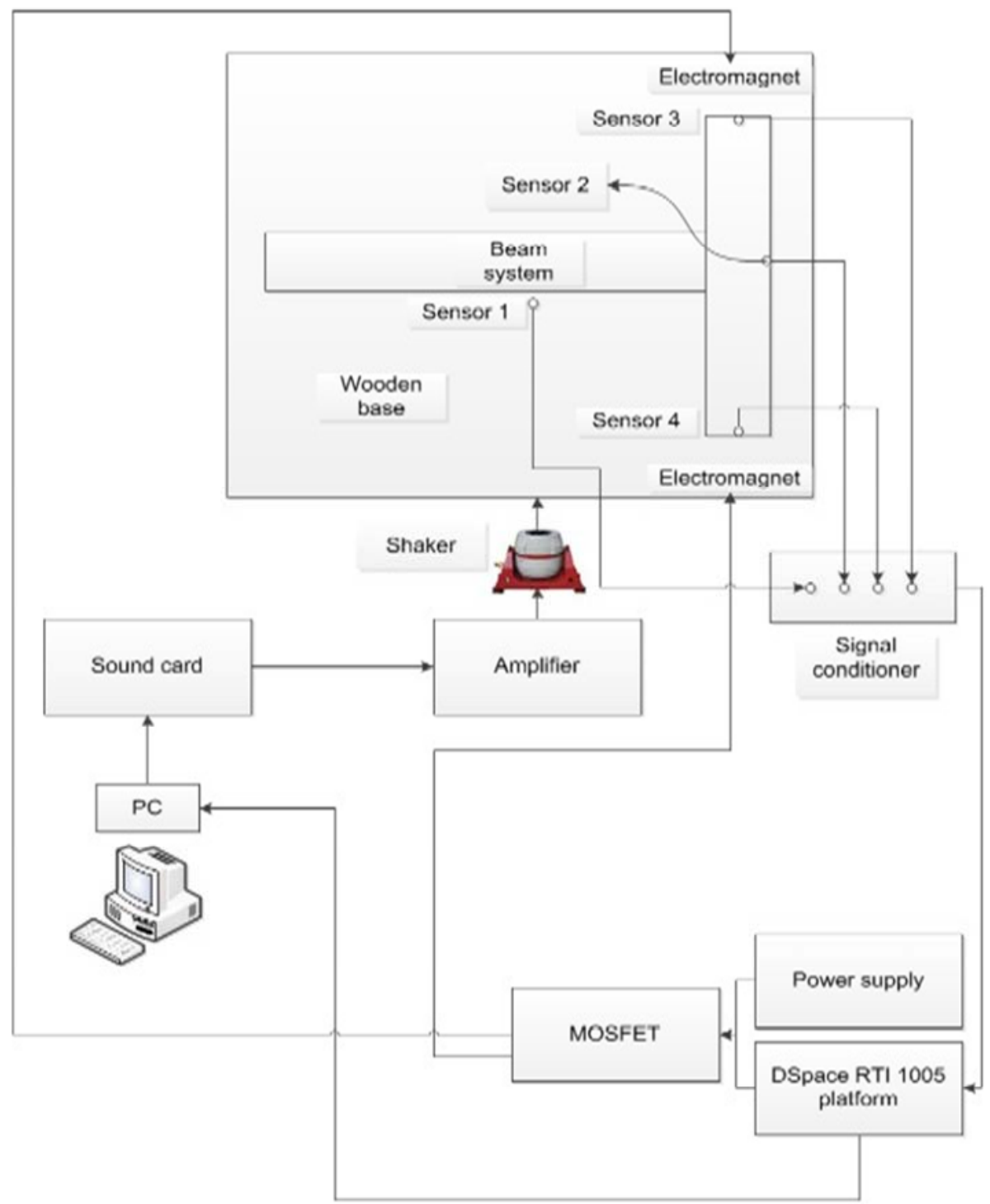

Figure 8. Schematic diagram of the setup used for the experiment to validate the response of the system with switchable friction.

\subsection{Time Response of the System with Switchable Friction}

Based on the estimated two degree of freedom model parameters determined for the test rig by Ismail and Ferguson [1], the system with a thin secondary beam can serve as the comparison to a two degree of freedom model with a low stiffness ratio. It is expected that it is possible to have a small reduction in the displacement at the end of the primary beam during the experiment and the primary mass acceleration would be smooth.

The measures used to perform control and the voltage generated by the D Space system to control the switchable electromagnet are shown in Figure 9 and Figure 10 respectively. The voltage switches between 0 and $10 \mathrm{~V}, 0 \mathrm{~V}$ means no voltage generated so friction is on and $10 \mathrm{~V}$ means the electromagnet attracts the steel beam resulting in zero friction.

The relative displacement changes sign at normalised time about 0.4 and 0.82 , while the relative velocity changes sign at normalised time about 0.36 and 0.73 . The absolute velocity at the end of the primary beam changes sign at normalised time about 0.57 . High frequency oscillations of small magnitude during the start of the motion (until normalised time about 0.1) were also observed that caused the logic parameters changing sign and switching several times during this time interval. Friction is supposed to switch off from the start of the base input since all control logic parameters act in the same direction. However, this could not be achieved during the experiment which shows the absolute velocity at the end of the primary beam and the relative velocity between the end of the secondary beam and the base changed sign very rapidly during that short period, resulting in friction switching. The high frequency vibration may be caused by the excitation of the higher order beam modes. False switching behaviour can be eliminated in the future by using a low pass filter to filter the high frequencies of the velocities 
or setting the threshold on the signal levels required for the switching. As the velocity response increases, the process of

switching improves and works as expected.

(a)

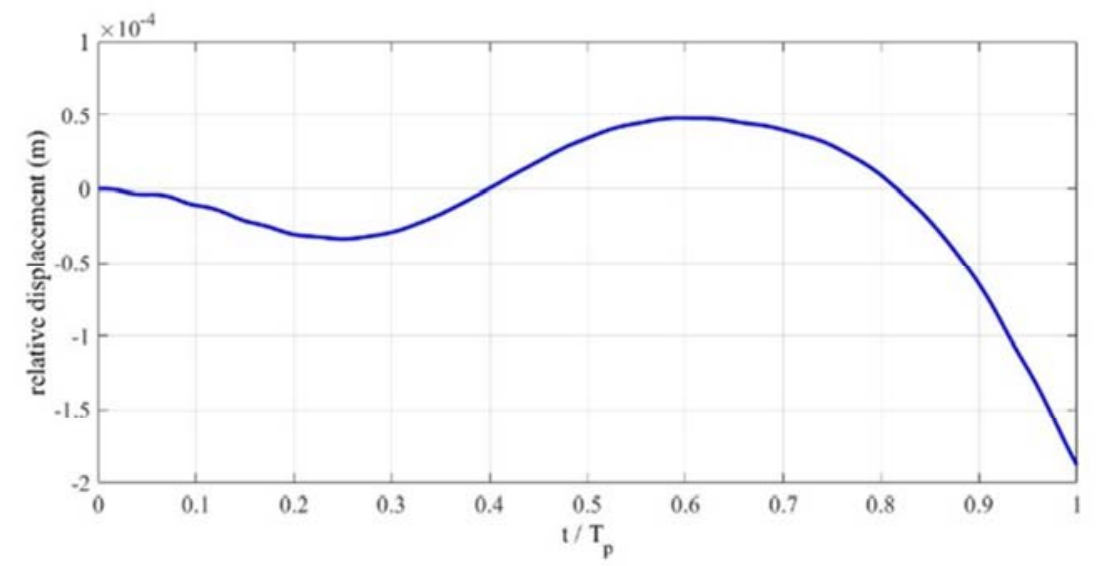

(b)

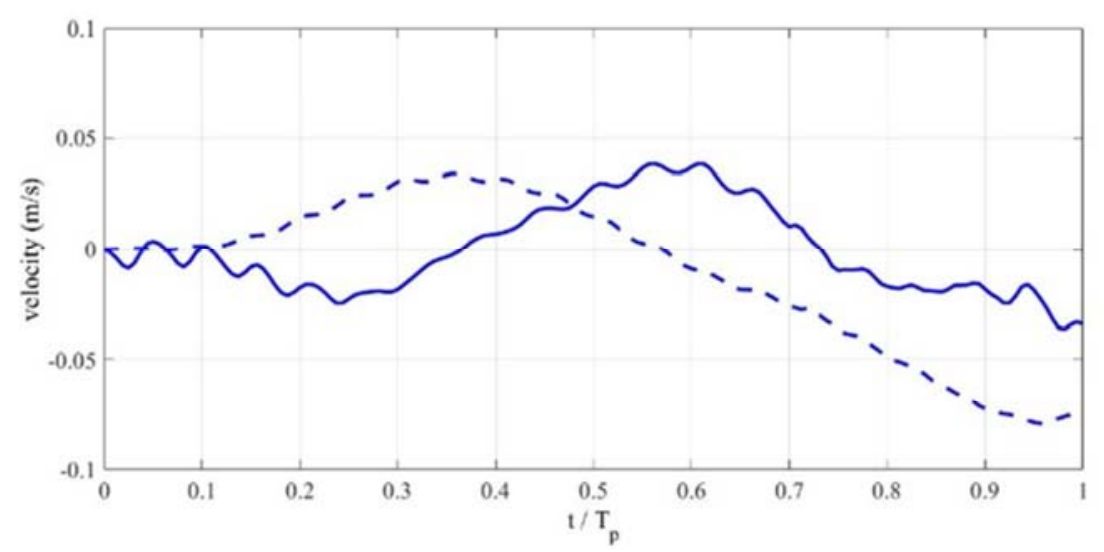

Figure 9. The parameters used to implement a control logic. (a) Measured relative displacement between the end of the primary mass and the base for the system with switchable friction. (b) Measured relative velocity between the end of the secondary beam and the base for the system with switchable friction. (c) Measured absolute velocity at the end of the primary beam for the system with switchable friction.

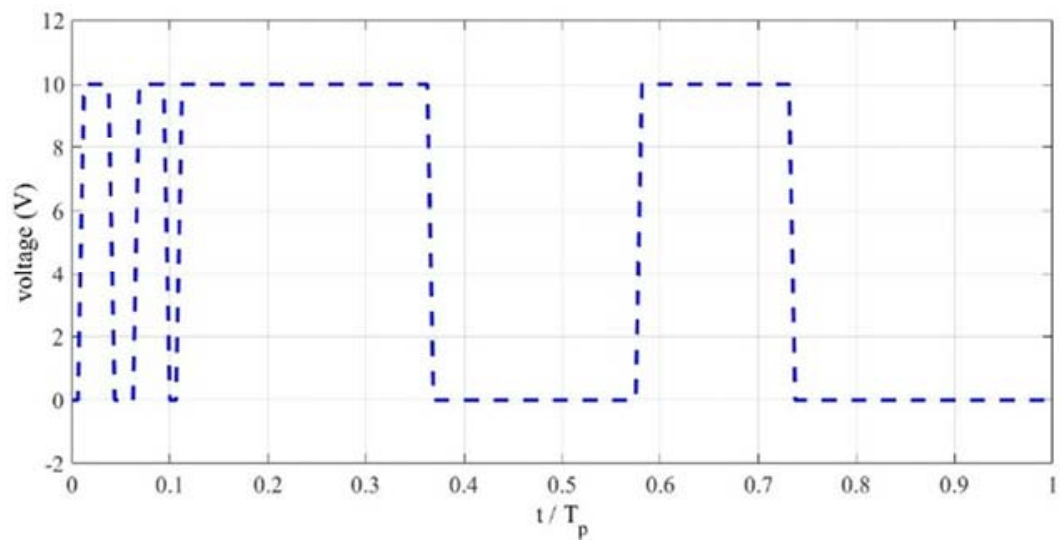

Figure 10. Output voltage versus time as the base input is applied to the beam system with switchable friction. When voltage=10 V the electromagnet attracts the steel beam and friction is off. When voltage $=0 \mathrm{~V}$ the electromagnet is off, the steel beam applies friction at the end of the secondary beam.

The normalised displacement is presented in Figure 11 for the case when friction is low and the secondary beam slides with respect to the friction interface. A similar pulse length to the passive case validation experiment conducted by Ismail and Ferguson (2017) was used. The displacement of the corresponding system is also shown. The switchable friction reduces the normalised primary beam displacement from 1.59 for the passive system to 1.33 .

There is not much difference between the switchable and passive system response at the start of the input as friction is 
not turned off completely. After the friction is switched off at the normalised time about 0.1 , the response of the switchable system starts to differ from the passive system. When the friction is switched on at the normalised time about 0.36 the displacement of the primary beam end reaches a smaller peak than the passive system reducing by approximately $16.4 \%$.

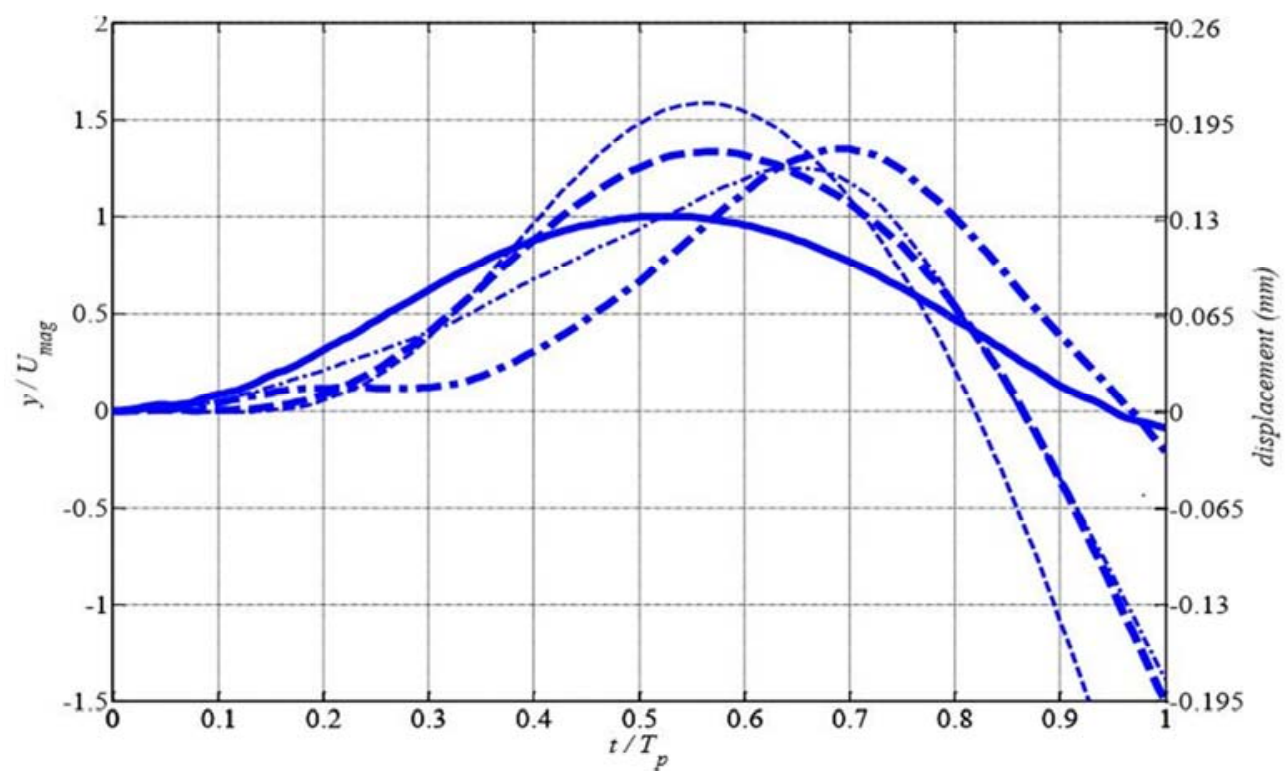

Figure 11. Comparison between measured displacement responses of the beam system with switchable and passive friction. Solid thick line: base input. Dash thick line: end of primary beam, switchable friction. Dash thin line: end of primary beam, passive friction. Dash dot thick line: end of secondary beam, switchable friction. Dash dot thin line: end of secondary beam, passive friction. Non-dimensional displacement, $y=\frac{\omega_{1}^{2} x}{g}$. Non-dimensional base input magnitude, $U_{\text {mag }}=\frac{\omega_{1}^{2} u_{m a g}}{g}$.

The comparison with the two degree of freedom model simulation is shown in Figure 12. Friction of amplitude $\hat{F}=$ 0.48 was used in the simulation based on the level estimated previously by Ismail and Ferguson (2017). There is slight difference in the measured and predicted responses. For the first half of the pulse duration, the difference is small. After that, the difference increases when the base starts to move in the negative direction (downward). The difference is caused by the difference in the base input compared to the assumed versed sine curve. The deviation is also caused by the process of rapid switching at the beginning of the base input or by non constant friction levels as the sliding occurs.

Table 3 compares the measured and predicted maximum displacement. The comparison shows good agreement except for the displacement at the end of the secondary beam for the case of switchable friction, which is about $18 \%$ lower than the prediction. This is expected since small changes in friction could cause significant change in the secondary mass response for a low stiffness ratio i.e. a soft secondary spring. The difference of $18 \%$ is considered acceptable since there could exist small changes in the level of friction as sliding occurs. The measured response at the end of the primary mass is very close to the predicted response, within $4 \%$ of the value.

(a)

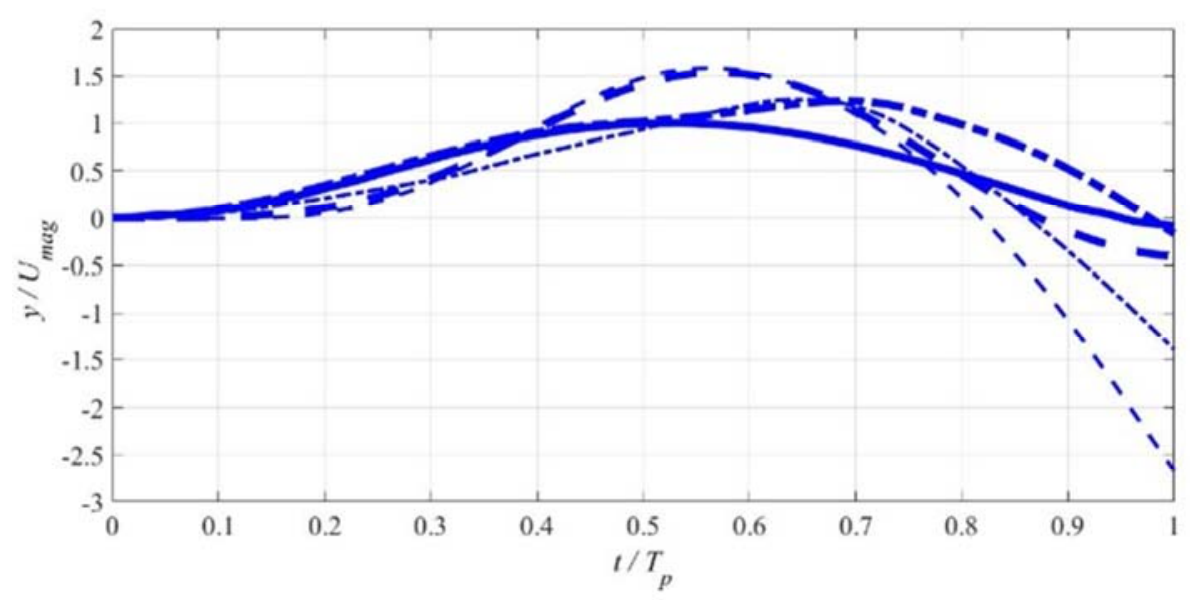


(b)

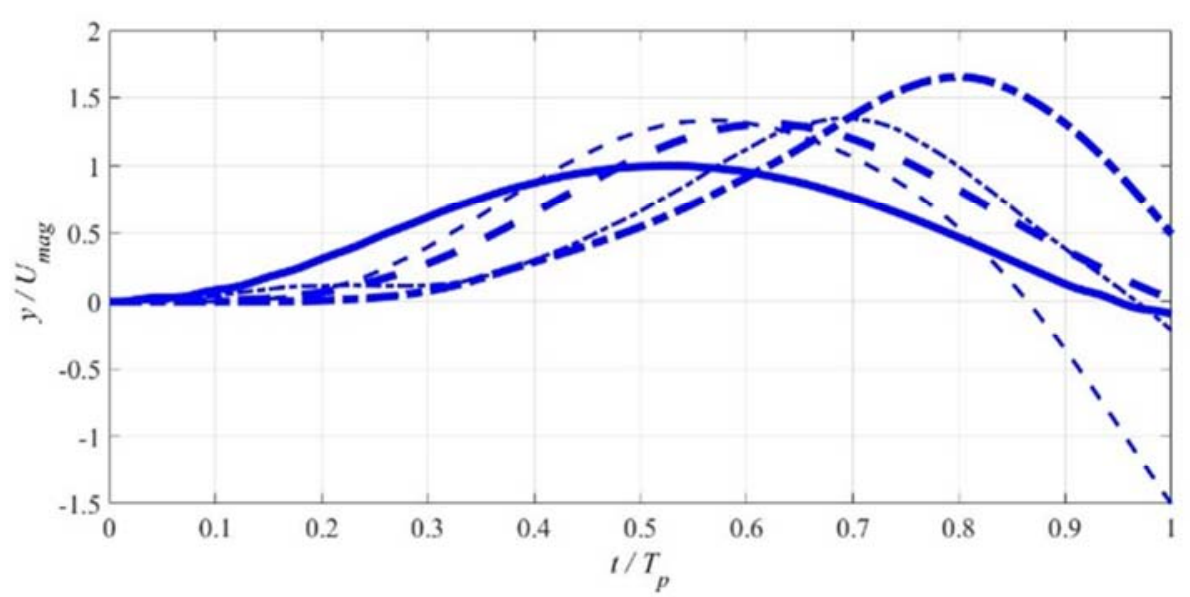

Figure 12. Comparison between measured displacement responses of the beam system with ODE simulation. Solid thick line: base input. Dash thick line: ODE simulation, primary mass. Dash thin line: measured, end of primary beam. Dash dot thick line: ODE simulation, secondary mass. Dash dot thin line: measured, end of secondary beam. (a) Passive friction. (b) Switchable friction. Non-dimensional displacement, $y=\frac{\omega_{1}^{2} x}{g}$. Non-dimensional base input magnitude, $U_{\text {mag }}=\frac{\omega_{1}^{2} u_{\text {mag }}}{g}$. Non-dimensional friction, $\hat{F}=0.48$.

Table 3. Comparison between the measured and ODE simulation maximum displacements. The maximum displacements are normalized with the maximum base input displacement. Non-dimensional friction, : $\hat{F}=0.48$.

\begin{tabular}{llll}
\hline \multirow{2}{*}{ Categories } & & Experiment & \\
\cline { 3 - 4 } & & End of primary beam & End of secondary beam \\
\hline \multirow{2}{*}{ Switchable friction } & Measured & 1.33 & 1.35 \\
& ODE simulation & 1.31 & 1.65 \\
\multirow{2}{*}{ Passive friction } & Measured & 1.59 & 1.25 \\
& ODE simulation & 1.54 & 1.23 \\
\hline
\end{tabular}

The reduction in the velocity response of the switchable system with the corresponding passive system is shown in Figure 13.

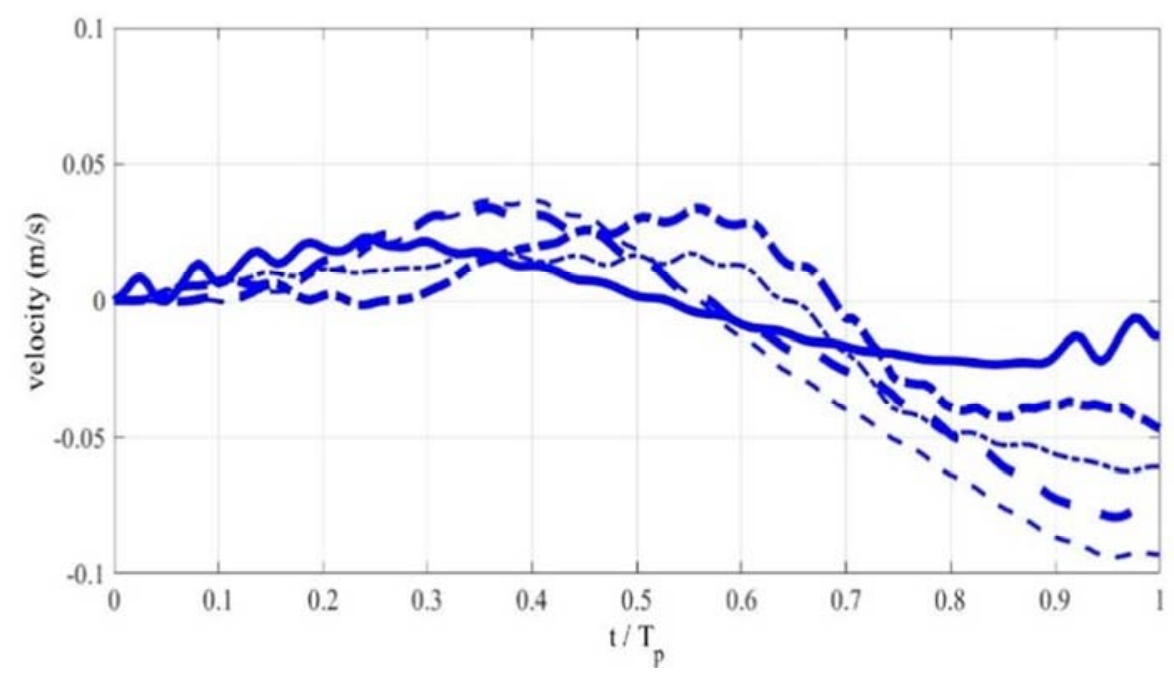

Figure 13. Comparison between measured velocity responses of the beam system with switchable and passive friction. Solid thick line: base input. Dash thick line: end of primary beam, switchable friction. Dash thin line: end of primary beam, passive friction. Dash dot thick line: end of secondary beam, switchable friction. Dash dot thin line: end of secondary beam, passive friction.

The acceleration of the switchable system with the corresponding passive system is shown in Figure 14. The acceleration at the end of the primary beam is much smoother than the secondary beam especially in the region of switching. This is as expected since the friction is applied directly to the secondary beam and caused discontinuous acceleration. Since the beam system is set to represent the two degree of freedom model with a soft secondary spring, the acceleration at the end of the primary beam remains relatively smooth. This being the main reason to improve the 
acceleration of the primary isolated mass.

(a)

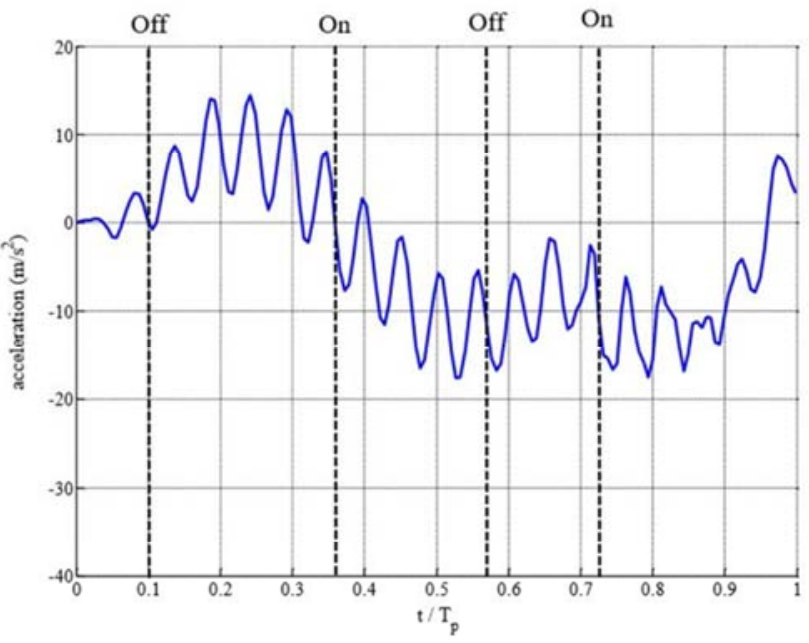

(b)

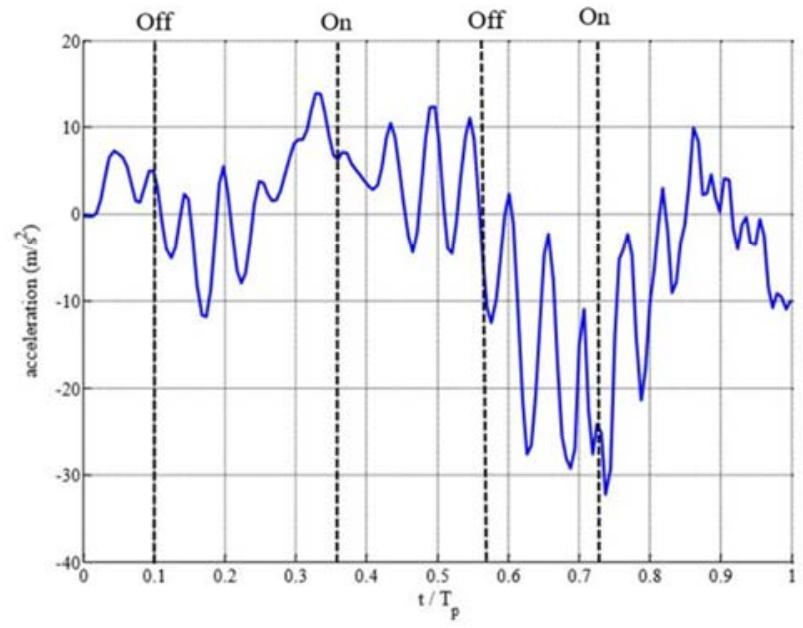

Figure 14. Measured acceleration response of the beam system with switchable friction. (a) End of the primary beam. (b) End of the secondary beam. The thick dashed lines mark the point of switching friction on and off.

\section{Conclusions}

Control strategies have been introduced for both a benchmark single and an alternative two degree of freedom system with Coulomb friction. Control strategies are outlined to switch on and off the friction according to the direction of the primary spring force, friction and primary mass velocity to reduce the net force acting on the primary mass and reduce the displacement. The response is evaluated only for the case of pulse length equal to the natural period of the system.

The single degree of freedom benchmark system produces a maximum displacement reduction up to $44 \%$ compared to the corresponding passive system when significantly large enough friction is applied. However, the acceleration experiences discontinuity when friction switches. A two degree of freedom system was then introduced and similar to the case of a single degree of freedom system, the displacement is smaller than the respective passive system only when friction is high. An intermediate stiffness ratio is enough to provide a maximum displacement smaller than the magnitude of the base input and at the same time provide a smooth acceleration especially at the point of switching onoff friction. A displacement reduction of up to $43 \%$ compared to the corresponding passive system is also achievable at higher stiffness ratios with the expected acceleration smoother than the single degree of freedom system.

The control of switching on and off friction was implemented by an electromagnet which works almost instantaneously in real time. The system behaved as expected, with only slight explainable differences. The switchable system gives a smaller peak displacement than the passive system at the end of a primary beam, which serves as a comparison to the primary mass in a two degree of freedom model. This shows that the switchable system provides an improvement to the shock response in comparison to a passive system. Furthermore, the acceleration at the end of the primary beam is very smooth. This shows that the two degree of freedom model, with friction applied to the secondary mass, provides a preferable acceleration of the primary targeted mass. By using switchable friction in the two degree of freedom model it is possible to obtain a better displacement and acceleration compared to a single degree of freedom system. The current experimental validation does not represent the best-case scenario, since this study was conducted just to see the fundamental characteristics of switchable friction and how it can be utilised.

\section{Acknowledgements}

The authors wish to express their gratitude to the Majlis Amanah Rakyat (MARA) for the financial support of the research programme reported herein.

\section{References}

[1] Ismail MI and Ferguson NS (2017) Passive Shock Isolation Utilising Dry Friction. Shock and Vibration vol. 2017, Article ID 7313809, 21 pages, 2017. doi: 10.1155/2017/7313809.

[2] Ismail MI (2012) Shock isolation systems incorporating Coulomb friction. PhD Thesis, University of Southampton, UK.

[3] Lu LY et al. (2010) Experiment and analysis of a fuzzy controlled piezoelectric seismic isolation system. Journal of Sound and Vibration 2010. 329: p. 1992-2014.

[4] Ramirez DFL (2008) Shock isolation using switchable stiffness. PhD Thesis, University of Southampton, UK.

[5] Liu Y, Waters TP and Brennan MJ (2005) A comparison of semi active damping control strategies for vibration isolation of harmonic disturbances. Journal of Sound and Vibration 2005. 280: p. 21-39.

[6] Jalili N (2002) A comparative study and analysis of semi active vibration control systems. Journal of Vibration and Acoustics 124: p. 593-605. 
[7] Karnopp DC, Crosby MJ and Harwood RA (1974) Vibration control using semi active force generators. Journal of Engineering for Industry, 1974. 96: p. 619-626.

[8] Dupont P, Kasturi P and Stokes A (1997) Semi active control of friction dampers. Journal of Sound and Vibration 202 (2): $p$ 203-218.

[9] Ferri AA and Heck BS (1992) Semi active suspension using dry friction energy dissipation. In: American Control Conference: Chicago, IL, p. 31-35.

[10] Yamaguchi H and Yashima M (1997) Vibration reduction and isolation performance for on off control of a friction force at a spring support. Journal of Sound and Vibration 1997. 208 (5): p. $729-743$.

[11] Stammers CW and Sireteanu T (1998) Vibration control of machines by use of semi active dry friction damping. Journal of Sound and Vibration 1998. 209 (4): p. 671684.

[12] Guglielmino E, Kevin AE and Ghigliazza R (2004) On the control of the friction force. Meccanica 2004. 39: p. 395-406.

[13] Ahmadian M, Brian R and Song X (2000) No jerk semi active skyhook control method and apparatus. United States Patent.

[14] Agrawal AK and Yang JN (2000) Semi active control strategies for buildings subject to near-field earthquakes. In: Smart Structures and Materials: Smart Systems for Bridges, Structures and Highways, Newport Beach, California, p. 359370 .

[15] Agrawal AK, Yang JN and He WL (2003) Applications of some semiactive control systems to benchmark cable-stayed bridge. Journal of Structural Engineering 129: p. 884-894.
[16] Jalili N and Ramaratnam A (2006) A switched stiffness approach for structural vibration control: theory and real time implementation. Journal of Sound and Vibration 2006. 291: p. 258-274.

[17] Ledezma-Ramírez DF and Tapia-González PE (2015) Experimental characterisation of dry friction isolators for shock vibration isolation. Proc. ICSV22, The 22nd International Congress on Sound and Vibration, Florence, Italy July 2015.

[18] Guzman-Nieto M, Tapia-González PE and Ledezma-Ramírez DF (2015) Low Frequency Experimental Analysis of Dry Friction Damping in Cable Isolators. Journal of Low Frequency Noise, Vibration and Active Control, vol. 34, 4: pp. 513-524. 2015.

[19] Tapia-González PE and Ledezma-Ramírez DF (2017) Experimental characterisation of dry friction isolators for shock and vibration. Journal of Low Frequency Noise, Vibration and Active Control vol. 36, 1: pp. 83-95. 2017.

[20] De Ni, Ru-peng Zhu, He-yun Bao, Feng-xia Lu and Qiu-ju Fu (2012) Influence of damping ratios on the dynamical characteristics of vibration isolation system based on Smart Spring Mechanism. Proc 3rd International Conference on System Science, Engineering Design and Manufacturing Informatization, Chengdu, China.

[21] Gustavo Rocha Vieira W, Nitzsche F and De Marqui Jr C (2017) The Use of Damping Based Semi-Active Control Algorithms in the Mechanical Smart-Spring System. Journal of Vibration and Acoustics 140 (2), 021011, 11 pages. 